\title{
The positive solutions of infinite-point boundary value problem of fractional differential equations on the infinite interval
}

\section{Xiaochen Li, Xiping Liu*, Mei Jia and Luchao Zhang}

"Correspondence:

xipingliu@usst.edu.cn

College of Science, University of

Shanghai for Science and

Technology, Shanghai, 200093,

China

\begin{abstract}
In this paper, we consider a class of infinite-point boundary value problems of fractional differential equations on the infinite interval $[0,+\infty)$ with a disturbance parameter. By using the method of upper and lower solutions, fixed point index theory and some fixed point theorems, the existence, multiplicity and nonexistence for the positive solution of the boundary value problem are obtained, respectively. The impact of the disturbance parameters on the existence of positive solutions is also given. Finally, some examples are presented to illustrate the wide range of potential applications of our main results.
\end{abstract}

Keywords: fractional differential equations; infinite-point boundary value problem; half line; positive solution; $L^{1}$-Carathéodory conditions

\section{Introduction}

In this paper, we are concerned with a class of infinite-point boundary value problems of fractional differential equations on the infinite interval with a disturbance parameter $\lambda$ as follows:

$$
\left\{\begin{array}{l}
D_{0^{+}}^{\delta} u(t)+q(t) f(t, u(t))=0, \quad t \in(0,+\infty) \\
u(0)=D_{0^{+}}^{\delta-1} u(+\infty)=0 \\
D_{0^{+}}^{\delta-2} u(0)=\sum_{i=1}^{\infty} g\left(\xi_{i}\right) D_{0^{+}}^{\delta-1} u\left(\xi_{i}\right)+\lambda
\end{array}\right.
$$

where $D_{0^{+}}^{\delta}$ is the standard Riemann-Liouville fractional derivative, $2<\delta<3.0<\xi_{1}<$ $\xi_{2}<\cdots<\xi_{i}<\cdots<+\infty, i=1,2, \ldots, g\left(\xi_{i}\right) \geq 0$ and $\sum_{i=1}^{\infty} g\left(\xi_{i}\right)$ is convergent. $\mathbb{R}^{+}=[0,+\infty)$, $f: \mathbb{R}^{+} \times \mathbb{R}^{+} \rightarrow \mathbb{R}^{+}$is an $L^{1}$-Carathéodory function, the disturbance parameter $\lambda \in \mathbb{R}^{+}$. $D_{0^{+}}^{\delta-1} u(+\infty):=\lim _{t \rightarrow+\infty} D_{0^{+}}^{\delta-1} u(t)$ exists.

In recent years, the theory of fractional differential equations has been widely used in various fields, such as physics, mechanics, chemistry, engineering, etc., see [1-4]. Meanwhile, the study of boundary value problems of fractional differential equations has gained plenty of meaningful results and has been growing rapidly, see [5-20].

(c) The Author(s) 2017. This article is distributed under the terms of the Creative Commons Attribution 4.0 International License (http://creativecommons.org/licenses/by/4.0/), which permits unrestricted use, distribution, and reproduction in any medium, provided you give appropriate credit to the original author(s) and the source, provide a link to the Creative Commons license, and indicate if changes were made. 
In [14], the authors studied the existence and nonexistence of the positive solutions for the fractional differential equation with two disturbance parameters

$$
\begin{cases}D_{0^{+}}^{\delta} u(t)+f(t, u(t))=0, & t \in(0,1) \\ \lim _{t \rightarrow 0^{+}} t^{2-\delta} u(t)=a, & u(1)=b\end{cases}
$$

where $1<\delta<2$, disturbance parameters $a \geq 0, b \geq 0$. Under certain conditions, the authors studied the impact of the disturbance parameters $a$ and $b$ on the existence of positive solutions.

As an important part of fractional differential equations, the boundary value problems on infinite intervals have also been extensively researched, see [21-27]. In [21], Liang and Zhang studied the following $m$-point boundary value problem of fractional differential equations on the infinite interval:

$$
\left\{\begin{array}{l}
D_{0^{+}}^{\delta} u(t)+a(t) f(u(t))=0, \quad t \in(0,+\infty), \\
u(0)=u^{\prime}(0)=0, \quad D_{0^{+}}^{\delta-1} u(+\infty)=\sum_{i=1}^{m-2} \gamma_{i} u\left(\xi_{i}\right),
\end{array}\right.
$$

where $2<\delta \leq 3,0<\xi_{1}<\xi_{2}<\cdots<\xi_{m-2}<+\infty, \gamma_{i} \geq 0, i=1,2, \ldots, m-2$ and $\sum_{i=1}^{m-2} \gamma_{i} \xi_{i}^{\delta-1}<$ $\Gamma(\delta)$. By using the Leggett-Williams fixed point theorem, the existence of three positive solutions for the boundary value problem on the infinite interval was obtained.

In [23], authors investigated the integral boundary value problem of fractional differential equations on infinite intervals with two disturbance parameters

$$
\left\{\begin{array}{l}
D_{0+}^{\delta} u(t)+f\left(t, u(t), D_{0+}^{\delta-1} u(t)\right)=0, \quad t \in(0,+\infty), \\
u(0)=0 \\
D_{0+}^{\delta-1} u(\infty)=\int_{0}^{\tau} g_{1}(s) u(s) \mathrm{d} s+a, \\
D_{0+}^{\delta-2} u(0)=\int_{0}^{\tau} g_{2}(s) u(s) \mathrm{d} s+b,
\end{array}\right.
$$

where $2<\delta \leq 3, f$ satisfies the $L^{1}$-Caratheódory conditions, $g_{1}, g_{2} \in L^{1}([0,+\infty))$ are nonnegative, disturbance parameters $a, b \in[0,+\infty)$.

The purpose of this paper is to investigate the existence of positive solutions for the infinite-point boundary value problem of fractional differential equations on the half-line (1.1). Moreover, the impact of the disturbance parameters on the existence and nonexistence of positive solutions is established. Finally, some examples are presented to illustrate the main results.

\section{Preliminaries}

For the convenience of the readers, we present here some basic definitions and lemmas, which are used throughout this paper.

The function $f: \mathbb{R}^{+} \times \mathbb{R}^{+} \rightarrow \mathbb{R}$ is called an $L^{1}$-Carathéodory function if

(1) for each $u \in \mathbb{R}^{+}, t \mapsto f(t, u)$ is measurable on $t \in \mathbb{R}^{+}$;

(2) for a.e. $t \in \mathbb{R}^{+}, u \mapsto f(t, u)$ is continuous on $u \in \mathbb{R}^{+}$;

(3) for each $r>0$, there exists $\varphi_{r} \in L^{1}\left(\mathbb{R}^{+}\right)$with $\varphi_{r}(t) \geq 0$ on $t \in \mathbb{R}^{+}$such that

$$
\left|f\left(t,\left(1+t^{\delta-1}\right) u\right)\right| \leq \varphi_{r}(t), \quad \text { for all }|u| \leq r \text {, and a.e. } t \in \mathbb{R}^{+} .
$$


Throughout this paper, we always assume that the following hypotheses hold:

(H1) $q \in L^{1}\left(\mathbb{R}^{+}\right)$is nonnegative and $\int_{0}^{+\infty} q(s) \varphi_{r}(s) \mathrm{d} s<+\infty$ for any $r>0$;

(H2) $f(t, u)$ is monotone increasing with respect to $u \in \mathbb{R}^{+}$for each $t \in \mathbb{R}^{+}$.

Definition 2.1 (see [1]) Let $p>0$. The Riemann-Liouville fractional integral of order $p$ of a function $u: \mathbb{R}^{+} \rightarrow \mathbb{R}$ is given by

$$
I_{0^{+}}^{p} u(t)=\frac{1}{\Gamma(p)} \int_{0}^{t}(t-s)^{p-1} u(s) \mathrm{d} s
$$

provided the integral exists.

Definition 2.2 (see [1]) Let $p>0$. The Riemann-Liouville fractional derivative of order $p$ of a function $u: \mathbb{R}^{+} \rightarrow \mathbb{R}$ is given by

$$
D_{0^{+}}^{p} u(t)=D^{n} I_{0^{+}}^{n-p} u(t)=\frac{1}{\Gamma(n-p)}\left(\frac{\mathrm{d}}{\mathrm{d} t}\right)^{n} \int_{0}^{t} \frac{u(s)}{(t-s)^{p-n+1}} \mathrm{~d} s,
$$

where $n$ is the smallest integer greater than or equal to $p$, provided the right-hand side is pointwise defined on $\mathbb{R}^{+}$.

Denote

$$
E=\left\{u \in C\left(\mathbb{R}^{+}\right): \sup _{t \in \mathbb{R}^{+}} \frac{|u(t)|}{1+t^{\delta-1}}<+\infty\right\},
$$

endowed with the norm $\|u\|=\sup _{t \in \mathbb{R}^{+}} \frac{|u(t)|}{1+t^{\delta-1}}$, then $E$ is a Banach space.

Definition 2.3 We say that $u=u(t)$ is a solution of boundary value problem (1.1), if $u \in E$, $D_{0^{+}}^{\delta} u \in L^{1}\left(\mathbb{R}^{+}\right)$and satisfies (1.1). Moreover, if $u(t) \geq 0, t \in \mathbb{R}^{+}$, we say that $u$ is a positive solution of boundary value problem (1.1).

Lemma 2.1 Suppose $h \in L^{1}\left(\mathbb{R}^{+}\right)$and $2<\delta<3$, then the following boundary value problem

$$
\left\{\begin{array}{l}
D_{0^{+}}^{\delta} u(t)+h(t)=0, \quad t \in(0,+\infty), \\
u(0)=D_{0^{+}}^{\delta-1} u(+\infty)=0 \\
D_{0^{+}}^{\delta-2} u(0)=\sum_{i=1}^{\infty} g\left(\xi_{i}\right) D_{0^{+}}^{\delta-1} u\left(\xi_{i}\right)+\lambda
\end{array}\right.
$$

has a unique solution

$$
u(t)=\int_{0}^{+\infty} G(t, s) h(s) \mathrm{d} s+\frac{\lambda t^{\delta-2}}{\Gamma(\delta-1)}
$$

where

$$
\begin{aligned}
& G(t, s)=G_{1}(t, s)+G_{2}(t, s), \\
& G_{1}(t, s)=\frac{1}{\Gamma(\delta)} \begin{cases}t^{\delta-1}-(t-s)^{\delta-1}, & 0 \leq s \leq t<+\infty, \\
t^{\delta-1}, & 0 \leq t<s<+\infty,\end{cases}
\end{aligned}
$$




$$
G_{2}(t, s)=\frac{1}{\Gamma(\delta-1)} \sum_{i=1}^{\infty} g\left(\xi_{i}\right) \chi\left(\xi_{i}, s\right) t^{\delta-2}
$$

and the characteristic function $\chi$ is defined by

$$
\chi\left(\xi_{i}, s\right)=\left\{\begin{array}{ll}
1, & s \geq \xi_{i}, \\
0, & s<\xi_{i},
\end{array} \quad i=1,2, \ldots .\right.
$$

Proof Since $D_{0+}^{\delta} u(t)+h(t)=0$, we have

$$
u(t)=-\frac{1}{\Gamma(\delta)} \int_{0}^{t}(t-s)^{\delta-1} h(s) \mathrm{d} s+c_{1} t^{\delta-1}+c_{2} t^{\delta-2}+c_{3} t^{\delta-3},
$$

where $c_{i} \in \mathbb{R}, i=1,2$, 3 .

Since $u(0)=0$, then $c_{3}=0$ and

$$
D_{0^{+}}^{\delta-1} u(t)=-\int_{0}^{t} h(s) \mathrm{d} s+c_{1} \Gamma(\delta), \quad D_{0^{+}}^{\delta-2} u(t)=-\int_{0}^{t}(t-s) h(s) \mathrm{d} s+c_{1} \Gamma(\delta) t+c_{2} \Gamma(\delta-1) .
$$

By the boundary conditions, we can get

$$
\left\{\begin{array}{l}
-\int_{0}^{+\infty} h(s) \mathrm{d} s+c_{1} \Gamma(\delta)=0, \\
c_{2} \Gamma(\delta-1)=\sum_{i=1}^{\infty} g\left(\xi_{i}\right) \int_{\xi_{i}}^{+\infty} h(s) \mathrm{d} s+\lambda,
\end{array}\right.
$$

and $c_{1}=\frac{1}{\Gamma(\delta)} \int_{0}^{+\infty} h(s) \mathrm{d} s, c_{2}=\frac{1}{\Gamma(\delta-1)} \sum_{i=1}^{\infty} g\left(\xi_{i}\right) \int_{\xi_{i}}^{+\infty} h(s) \mathrm{d} s+\frac{\lambda}{\Gamma(\delta-1)}$.

So

$$
\begin{aligned}
u(t)= & -\frac{1}{\Gamma(\delta)} \int_{0}^{t}(t-s)^{\delta-1} h(s) \mathrm{d} s+\frac{1}{\Gamma(\delta)} \int_{0}^{+\infty} t^{\delta-1} h(s) \mathrm{d} s \\
& +\frac{t^{\delta-2}}{\Gamma(\delta-1)} \sum_{i=1}^{\infty} g\left(\xi_{i}\right) \int_{\xi_{i}}^{+\infty} h(s) \mathrm{d} s+\frac{\lambda t^{\delta-2}}{\Gamma(\delta-1)} \\
= & \int_{0}^{+\infty} G_{1}(t, s) h(s) \mathrm{d} s+\frac{\sum_{i=1}^{\infty} g\left(\xi_{i}\right) t^{\delta-2}}{\Gamma(\delta-1)} \int_{0}^{+\infty} \chi\left(\xi_{i}, s\right) h(s) \mathrm{d} s+\frac{\lambda t^{\delta-2}}{\Gamma(\delta-1)} \\
= & \int_{0}^{+\infty} G_{1}(t, s) h(s) \mathrm{d} s+\int_{0}^{+\infty} G_{2}(t, s) h(s) \mathrm{d} s+\frac{\lambda t^{\delta-2}}{\Gamma(\delta-1)} \\
= & \int_{0}^{+\infty} G(t, s) h(s) \mathrm{d} s+\frac{\lambda t^{\delta-2}}{\Gamma(\delta-1)} .
\end{aligned}
$$

On the other hand, if $u$ satisfies (2.2), we can easily show that $u$ satisfies (2.1) and $D_{0^{+}}^{\delta} u \in$ $L^{1}\left(\mathbb{R}^{+}\right)$.

The proof is completed.

Lemma 2.2 Let $G(t, s), G_{1}(t, s)$ defined by (2.3) and (2.4) satisfy the following properties:

(1) $G(t, s), G_{1}(t, s) \geq 0$ for any $(t, s) \in \mathbb{R}^{+} \times \mathbb{R}^{+}$;

(2) $G_{1}(t, s)$ and $\frac{G_{1}(t, s)}{1+t^{\delta-1}}$ are continuous on $(t, s) \in \mathbb{R}^{+} \times \mathbb{R}^{+}$;

(3) $0 \leq \frac{G(t, s)}{1+t^{\delta-1}} \leq \frac{L}{\Gamma(\delta)}$ for any $t, s \in \mathbb{R}^{+}$, where the constant $L=1+(\delta-1) \sum_{i=1}^{\infty} g\left(\xi_{i}\right)$; 
(4) for a constant $k>1$, we have

$$
\min _{\frac{1}{k} \leq t \leq k} \frac{G_{1}(t, s)}{1+t^{\delta-1}} \geq \frac{1}{4 k^{2}\left(1+k^{\delta-1}\right)} \sup _{t \in \mathbb{R}^{+}} \frac{G_{1}(t, s)}{1+t^{\delta-1}}
$$

and

$$
\min _{\frac{1}{k} \leq t \leq k} \frac{G(t, s)}{1+t^{\delta-1}} \geq \frac{1}{4 k^{2}\left(1+k^{\delta-1}\right)} \sup _{t \in \mathbb{R}^{+}} \frac{G(t, s)}{1+t^{\delta-1}} .
$$

Proof By (2.4) and (2.5), the definitions of $G(t, s)$ and $G_{1}(t, s)$, the results (1) and (2) can be easily obtained.

(3) According to the definition of $G(t, s)$, we have

$$
\frac{G(t, s)}{1+t^{\delta-1}}=\frac{G_{1}(t, s)}{1+t^{\delta-1}}+\frac{G_{2}(t, s)}{1+t^{\delta-1}} \leq \frac{1}{\Gamma(\delta)}+\frac{1}{\Gamma(\delta-1)} \sum_{i=1}^{\infty} g\left(\xi_{i}\right)=\frac{L}{\Gamma(\delta)} .
$$

(4) The first inequality can be found in [21]

We can easily show that $\min _{\frac{1}{k} \leq t \leq k}\left\{\frac{t^{\delta-2}}{1+t^{\delta-1}}\right\}=\frac{k^{\delta-2}}{1+k^{\delta-1}}$ for $k>1$. Thus

$$
\begin{aligned}
\min _{\frac{1}{k} \leq t \leq k} \frac{G_{2}(t, s)}{1+t^{\delta-1}} & =\frac{1}{\Gamma(\delta-1)} \sum_{i=1}^{\infty} g\left(\xi_{i}\right) \chi\left(\xi_{i}, s\right) \min _{\frac{1}{k} \leq t \leq k} \frac{t^{\delta-2}}{1+t^{\delta-1}} \\
& =\frac{1}{\Gamma(\delta-1)} \sum_{i=1}^{\infty} g\left(\xi_{i}\right) \chi\left(\xi_{i}, s\right) \frac{k^{\delta-2}}{1+k^{\delta-1}} \\
& >\frac{1}{\Gamma(\delta-1)} \sum_{i=1}^{\infty} g\left(\xi_{i}\right) \chi\left(\xi_{i}, s\right) \frac{1}{1+k^{\delta-1}} \\
& >\frac{1}{1+k^{\delta-1}} \frac{1}{\Gamma(\delta-1)} \sum_{i=1}^{\infty} g\left(\xi_{i}\right) \chi\left(\xi_{i}, s\right) \sup _{t \in \mathbb{R}^{+}} \frac{t^{\delta-2}}{1+t^{\delta-1}} \\
& =\frac{1}{1+k^{\delta-1}} \sup _{t \in \mathbb{R}^{+}} \frac{G_{2}(t, s)}{1+t^{\delta-1}} .
\end{aligned}
$$

So

$$
\begin{aligned}
\min _{\frac{1}{k} \leq t \leq k} \frac{G(t, s)}{1+t^{\delta-1}} & \geq \min _{\frac{1}{k} \leq t \leq k} \frac{G_{1}(t, s)}{1+t^{\delta-1}}+\min _{\frac{1}{k} \leq t \leq k} \frac{G_{2}(t, s)}{1+t^{\delta-1}} \\
& \geq \frac{1}{4 k^{2}\left(1+k^{\delta-1}\right)} \sup _{t \in \mathbb{R}^{+}} \frac{G_{1}(t, s)}{1+t^{\delta-1}}+\frac{1}{1+k^{\delta-1}} \sup _{t \in \mathbb{R}^{+}} \frac{G_{2}(t, s)}{1+t^{\delta-1}} \\
& \geq \frac{1}{4 k^{2}\left(1+k^{\delta-1}\right)} \sup _{t \in \mathbb{R}^{+}} \frac{G(t, s)}{1+t^{\delta-1}} .
\end{aligned}
$$

Let

$$
P=\left\{u \in E: u(t) \geq 0, t \in \mathbb{R}^{+}\right\} .
$$

Then $P \subset E$ is a cone. 
For $u \in P$, let

$$
(T u)(t)=\int_{0}^{+\infty} G(t, s) q(s) f(s, u(s)) \mathrm{d} s+\frac{\lambda t^{\delta-2}}{\Gamma(\delta-1)} .
$$

Then $T: P \rightarrow E$.

Lemma 2.3 (see [24]) Let $V=\{u \in E:\|u\| \leq l\}, l>0, V_{1}=\left\{v=\frac{u}{1+t^{\delta-1}}: u \in V\right\}$. If $V_{1}$ is equicontinuous on any compact interval of $\mathbb{R}^{+}$and equiconvergent at infinity, then $V$ is relatively compact on $E$.

Lemma 2.4 Assume $(\mathrm{H} 1)$ holds, then $T: P \rightarrow P$ is completely continuous.

Proof For $u \in P \subset E$, since $\sup _{t \in \mathbb{R}^{+}} \frac{u(t)}{1+t^{\delta-1}}<+\infty$, there exists a constant $l>0$ such that $\|u\| \leq l$. Then $\frac{T u(t)}{1+t^{\delta-1}}<\frac{L}{\Gamma(\delta)} \int_{0}^{+\infty} q(s) \varphi_{l}(s) \mathrm{d} s+\frac{\lambda}{\Gamma(\delta-1)}<+\infty$, and $\operatorname{Tu}(t)$ is continuous with respect to $t \in \mathbb{R}^{+}$. So $T u \in E$ and $T: P \rightarrow E$ is well defined. Since $G, f, q$ are nonnegative, then $T u(t) \geq 0$, which implies $T u \in P$ for any $u \in P$.

(i) Let $\left\{u_{n}\right\} \subset P, u \in P$ such that $\left\|u_{n}-u\right\| \rightarrow 0$ as $n \rightarrow+\infty$, that is, $\frac{u_{n}(t)}{1+t^{\delta-1}} \rightarrow \frac{u(t)}{1+t^{\delta-1}}$. Then there exists a constant $r>0$ such that $\left\|u_{n}\right\| \leq r,\|u\| \leq r$. Since $f$ satisfies the $L^{1}$ Carathéodory conditions for a.e. $s \in \mathbb{R}^{+}$, then

$$
\left|f\left(s, u_{n}(s)\right)-f(s, u(s))\right| \rightarrow 0, \quad \text { as } n \rightarrow+\infty,
$$

and

$$
\left|f\left(s, u_{n}(s)\right)-f(s, u(s))\right|=\left|f\left(s,\left(1+s^{\delta-1}\right) \frac{u_{n}(s)}{1+s^{\delta-1}}\right)-f\left(s,\left(1+s^{\delta-1}\right) \frac{u(s)}{1+s^{\delta-1}}\right)\right| \leq 2 \varphi_{r}(s) .
$$

By the Lebesgue dominated convergence theorem,

$$
\int_{0}^{+\infty} q(s)\left|f\left(s, u_{n}(s)\right)-f(s, u(s))\right| \mathrm{d} s \rightarrow 0, \quad \text { as } n \rightarrow+\infty
$$

Therefore,

$$
\begin{aligned}
\frac{\left|T u_{n}(t)-T u(t)\right|}{1+t^{\delta-1}} & =\left|\int_{0}^{+\infty} \frac{G(t, s)}{1+t^{\delta-1}} q(s)\left(f\left(s, u_{n}(s)\right)-f(s, u(s))\right) \mathrm{d} s\right| \\
& \leq \int_{0}^{+\infty} \frac{G(t, s)}{1+t^{\delta-1}} q(s)\left|f\left(s, u_{n}(s)\right)-f(s, u(s))\right| \mathrm{d} s \\
& \leq \frac{L}{\Gamma(\delta)} \int_{0}^{+\infty} q(s)\left|f\left(s, u_{n}(s)\right)-f(s, u(s))\right| \mathrm{d} s \rightarrow 0, \quad \text { as } n \rightarrow+\infty .
\end{aligned}
$$

Hence, $\left\|T u_{n}-T u\right\| \rightarrow 0$ as $n \rightarrow+\infty$, which implies that $T$ is a continuous operator.

Let $B \subset P$ be a nonempty bounded closed subset. There exists a constant $l_{B}>0$ such that $\|u\| \leq l_{B}$ for all $u \in B$, and there exists $\varphi_{l_{B}} \in L^{1}\left(\mathbb{R}^{+}\right)$such that

$$
f(s, u(s))=f\left(s,\left(1+s^{\delta-1}\right) \frac{u(s)}{1+s^{\delta-1}}\right) \leq \varphi_{l_{B}}(s)
$$


For any $u \in B$,

$$
\begin{aligned}
\left|\frac{T u(t)}{1+t^{\delta-1}}\right| & =\left|\int_{0}^{+\infty} \frac{G(t, s)}{1+t^{\delta-1}} q(s) f(s, u(s)) \mathrm{d} s+\frac{\lambda}{\Gamma(\delta-1)} \frac{t^{\delta-2}}{1+t^{\delta-1}}\right| \\
& \leq \frac{L}{\Gamma(\delta)} \int_{0}^{+\infty} q(s) \varphi_{l_{B}}(s) \mathrm{d} s+\frac{\lambda}{\Gamma(\delta-1)}<+\infty .
\end{aligned}
$$

So, $\|T u\|<+\infty$. Then $T(B)$ is bounded and $T$ is uniformly bounded.

(ii) For any $T_{0}>0$, let $I=\left[0, T_{0}\right]$ be a compact interval. Because $\frac{G_{1}(t, s)}{1+t^{\delta-1}}$ is continuous for $(t, s) \in I \times I, \frac{t^{\delta-1}}{1+t^{\delta-1}}, \frac{t^{\delta-2}}{1+t^{\delta-1}}$ are continuous for $t \in I$, then they are uniformly continuous. So, for any $\varepsilon>0$, there exists a constant $0<\delta_{1}<\varepsilon$ such that

$$
\begin{aligned}
& \left|\frac{G_{1}\left(t_{1}, s_{1}\right)}{1+t_{1}^{\delta-1}}-\frac{G_{1}\left(t_{2}, s_{2}\right)}{1+t_{2}^{\delta-1}}\right|<\frac{\varepsilon}{\Gamma(\delta)}, \\
& \left|\frac{t_{1}^{\delta-1}}{1+t_{1}^{\delta-1}}-\frac{t_{2}^{\delta-1}}{1+t_{2}^{\delta-1}}\right|<\varepsilon, \quad\left|\frac{t_{1}^{\delta-2}}{1+t_{1}^{\delta-1}}-\frac{t_{2}^{\delta-2}}{1+t_{2}^{\delta-1}}\right|<\varepsilon
\end{aligned}
$$

for all $t_{1}, t_{2}, s_{1}, s_{2} \in I$ and $\left|t_{1}-t_{2}\right|<\delta_{1},\left|s_{1}-s_{2}\right|<\delta_{1}$.

From the definition of $G_{1}(t, s)$, for $s>t$,

$$
\left|\frac{G_{1}\left(t_{1}, s\right)}{1+t_{1}^{\delta-1}}-\frac{G_{1}\left(t_{2}, s\right)}{1+t_{2}^{\delta-1}}\right| \leq \frac{1}{\Gamma(\delta)}\left|\frac{t_{1}^{\delta-1}}{1+t_{1}^{\delta-1}}-\frac{t_{2}^{\delta-1}}{1+t_{2}^{\delta-1}}\right|<\frac{1}{\Gamma(\delta)} \varepsilon .
$$

Similarly, we can get

$$
\left|\frac{G_{2}\left(t_{1}, s\right)}{1+t_{1}^{\delta-1}}-\frac{G_{2}\left(t_{2}, s\right)}{1+t_{2}^{\delta-1}}\right| \leq \frac{1}{\Gamma(\delta-1)} \sum_{i=1}^{\infty} g\left(\xi_{i}\right)\left|\frac{t_{1}^{\delta-2}}{1+t_{1}^{\delta-1}}-\frac{t_{2}^{\delta-2}}{1+t_{2}^{\delta-1}}\right|<\frac{\varepsilon}{\Gamma(\delta-1)} \sum_{i=1}^{\infty} g\left(\xi_{i}\right) .
$$

Then, for each $u \in B$,

$$
\begin{aligned}
\left|\frac{T u\left(t_{1}\right)}{1+} t_{1}^{\delta-1}-\frac{T u\left(t_{2}\right)}{1+t_{2}^{\delta-1}}\right| & \int_{0}^{+\infty}\left|\frac{G_{1}\left(t_{1}, s\right)}{1+t_{1}^{\delta-1}}-\frac{G_{1}\left(t_{2}, s\right)}{1+t_{2}^{\delta-1}}\right| q(s)|f(s, u(s))| \mathrm{d} s \\
& +\int_{0}^{+\infty}\left|\frac{G_{2}\left(t_{1}, s\right)}{1+t_{1}^{\delta-1}}-\frac{G_{2}\left(t_{2}, s\right)}{1+t_{2}^{\delta-1}}\right| q(s)|f(s, u(s))| \mathrm{d} s+\frac{\lambda}{\Gamma(\delta-1)}\left|\frac{t_{1}^{\delta-2}}{1+t_{1}^{\delta-1}}-\frac{t_{2}^{\delta-2}}{1+t_{2}^{\delta-1}}\right| \\
\leq & \int_{0}^{T_{0}}\left|\frac{G_{1}\left(t_{1}, s\right)}{1+t_{1}^{\delta-1}}-\frac{G_{1}\left(t_{2}, s\right)}{1+t_{2}^{\delta-1}}\right| q(s)|f(s, u(s))| \mathrm{d} s \\
& +\int_{T_{0}}^{+\infty}\left|\frac{G_{1}\left(t_{1}, s\right)}{1+t_{1}^{\delta-1}}-\frac{G_{1}\left(t_{2}, s\right)}{1+t_{2}^{\delta-1}}\right| q(s)|f(s, u(s))| \mathrm{d} s \\
& +\frac{\varepsilon}{\Gamma(\delta-1)} \sum_{i=1}^{\infty} g\left(\xi_{i}\right) \int_{0}^{+\infty} q(s) f(s, u(s)) \mathrm{d} s+\frac{\lambda}{\Gamma(\delta-1)} \varepsilon \\
\leq & \frac{\varepsilon}{\Gamma(\delta)} \int_{0}^{T_{0}} q(s) \varphi_{l_{B}}(s) \mathrm{d} s+\frac{\varepsilon}{\Gamma(\delta)} \int_{T_{0}}^{+\infty} q(s) \varphi_{l_{B}}(s) \mathrm{d} s \\
& +\frac{\varepsilon}{\Gamma(\delta-1)} \sum_{i=1}^{\infty} g\left(\xi_{i}\right) \int_{0}^{+\infty} q(s) \varphi_{l_{B}}(s) \mathrm{d} s+\frac{\lambda}{\Gamma(\delta-1)} \varepsilon
\end{aligned}
$$




$$
\begin{aligned}
& \leq\left(\frac{1}{\Gamma(\delta)} \int_{0}^{+\infty} q(s) \varphi_{l_{B}}(s) \mathrm{d} s+\frac{1}{\Gamma(\delta-1)} \sum_{i=1}^{\infty} g\left(\xi_{i}\right) \int_{0}^{+\infty} q(s) \varphi_{l_{B}}(s) \mathrm{d} s+\frac{\lambda}{\Gamma(\delta-1)}\right) \varepsilon \\
& =\left(\frac{L}{\Gamma(\delta)} \int_{0}^{+\infty} q(s) \varphi_{l_{B}}(s) \mathrm{d} s+\frac{\lambda}{\Gamma(\delta-1)}\right) \varepsilon
\end{aligned}
$$

Therefore, $\frac{T u(t)}{1+t^{\delta-1}}$ is equicontinuous on $I$.

(iii) We prove that $T: P \rightarrow P$ is equiconvergent at $t=+\infty$. Since $\lim _{t \rightarrow+\infty} \frac{t^{\delta-1}}{1+t^{\delta-1}}=$ 1 and $\lim _{t \rightarrow+\infty} \frac{t^{\delta-2}}{1+t^{\delta-1}}=0$, then $\lim _{t \rightarrow+\infty} \frac{G_{1}(t, s)}{1+t^{\delta-1}}=0$ and $\lim _{t \rightarrow+\infty} \frac{G_{2}(t, s)}{1+t^{\delta-1}}=0$. Therefore $\lim _{t \rightarrow+\infty} \frac{G(t, s)}{1+t^{\delta-1}}=0$.

For any $u \in B$, we have

$$
\int_{0}^{+\infty} q(s) f(s, u(s)) \mathrm{d} s \leq \int_{0}^{+\infty} q(s) \varphi_{l_{B}}(s) \mathrm{d} s<+\infty
$$

and

$$
\lim _{t \rightarrow+\infty}\left|\frac{T u(t)}{1+t^{\delta-1}}\right|=\lim _{t \rightarrow+\infty}\left|\int_{0}^{+\infty} \frac{G(t, s)}{1+t^{\delta-1}} q(s) f(s, u(s)) \mathrm{d} s+\frac{\lambda}{\Gamma(\delta-1)} \frac{t^{\delta-2}}{1+t^{\delta-1}}\right|=0<+\infty .
$$

Hence, $T(B)$ is equiconvergent at infinity. Consequently, in view of Lemma 2.3, $T(B)$ is relatively compact, thus $T$ is a compact operator. So $T$ is completely continuous and the proof is finished.

Lemma 2.5 If boundary value problem (1.1) has a positive solution $u$, then for $t \in \mathbb{R}^{+}$,

$$
\min _{\frac{1}{k} \leq t \leq k} \frac{u(t)}{1+t^{\delta-1}} \geq \frac{1}{4 k^{2}\left(1+k^{\delta-1}\right)}\|u\| .
$$

Proof By Lemma 2.1, we have

$$
u(t)=\int_{0}^{+\infty} G(t, s) q(s) f(s, u(s)) \mathrm{d} s+\frac{\lambda}{\Gamma(\delta-1)} t^{\delta-2} .
$$

Then, according to (4) of Lemma 2.2, for $t \in \mathbb{R}^{+}$,

$$
\begin{aligned}
\min _{\frac{1}{k} \leq t \leq k} \frac{u(t)}{1+t^{\delta-1}} & =\min _{\frac{1}{k} \leq t \leq k}\left(\int_{0}^{+\infty} \frac{G(t, s)}{1+t^{\delta-1}} q(s) f(s, u(s)) \mathrm{d} s+\frac{\lambda}{\Gamma(\delta-1)} \frac{t^{\delta-2}}{1+t^{\delta-1}}\right) \\
& \geq \min _{\frac{1}{k} \leq t \leq k} \int_{0}^{+\infty} \frac{G(t, s)}{1+t^{\delta-1}} q(s) f(s, u(s)) \mathrm{d} s+\frac{\lambda}{\Gamma(\delta-1)} \min _{\frac{1}{k} \leq t \leq k} \frac{t^{\delta-2}}{1+t^{\delta-1}} \\
& \geq \frac{1}{4 k^{2}\left(1+k^{\delta-1}\right)} \int_{0}^{+\infty} \sup _{t \in \mathbb{R}^{+}} \frac{G(t, s)}{1+t^{\delta-1}} q(s) f(s, u(s)) \mathrm{d} s+\frac{\lambda}{\Gamma(\delta-1)} \frac{k^{\delta-2}}{1+k^{\delta-1}} \\
& \geq \frac{1}{4 k^{2}\left(1+k^{\delta-1}\right)} \sup _{t \in \mathbb{R}^{+}} \int_{0}^{+\infty} \frac{G(t, s)}{1+t^{\delta-1}} q(s) f(s, u(s)) \mathrm{d} s+\frac{\lambda}{\Gamma(\delta-1)} \frac{1}{1+k^{\delta-1}} \\
& \geq \frac{1}{4 k^{2}\left(1+k^{\delta-1}\right)} \sup _{t \in \mathbb{R}^{+}}\left(\int_{0}^{+\infty} \frac{G(t, s)}{1+t^{\delta-1}} q(s) f(s, u(s)) \mathrm{d} s+\frac{\lambda}{\Gamma(\delta-1)} \frac{t^{\delta-2}}{1+t^{\delta-1}}\right) \\
& =\frac{1}{4 k^{2}\left(1+k^{\delta-1}\right)}\|u\| .
\end{aligned}
$$

The proof is finished. 
By Lemma 2.1, we can easily show that the following lemma holds.

Lemma 2.6 Assume $u \in E, D_{0^{+}}^{\delta} u \in L^{1}\left(\mathbb{R}^{+}\right)$. Then boundary value problem (1.1) has a positive solution if and only if the operator $T$ has a fixed point in $P$.

Lemma 2.7 (see [28]) Let $P$ be a cone of the Banach space $E, \Omega \subset E$ be a bounded open set and $\theta \in \Omega$. Suppose $T: P \cap \bar{\Omega} \rightarrow P$ is a completely continuous operator. If $u \neq \mu$ Tu for any $u \in P \cap \partial \Omega$ and $\mu \in[0,1]$, then $i(T, P \cap \Omega, P)=1$.

\section{Comparison principle}

Definition 3.1 Let $\alpha \in E, D_{0^{+}}^{\delta} \alpha \in L^{1}\left(\mathbb{R}^{+}\right)$, we say that $\alpha=\alpha(t)$ is a lower solution of boundary value problem (1.1) if $\alpha$ satisfies

$$
\left\{\begin{array}{l}
-D_{0^{+}}^{\delta} \alpha(t) \leq q(t) f(t, \alpha(t)), \quad t \in(0,+\infty) \\
\alpha(0)=0, \quad D_{0^{+}}^{\delta-1} \alpha(+\infty)=0 \\
D_{0^{+}}^{\delta-2} \alpha(0) \leq \sum_{i=1}^{\infty} g\left(\xi_{i}\right) D_{0^{+}}^{\delta-1} u\left(\xi_{i}\right)+\lambda
\end{array}\right.
$$

Let $\beta \in E, D_{0^{+}}^{\delta} \beta \in L^{1}\left(\mathbb{R}^{+}\right)$, we say $\beta=\beta(t)$ is an upper solution of boundary value problem (1.1) if $\beta$ satisfies

$$
\left\{\begin{array}{l}
-D_{0^{+}}^{\delta} \beta(t) \geq q(t) f(t, \beta(t)), \quad t \in(0,+\infty) \\
\beta(0)=0, \quad D_{0^{+}}^{\delta-1} \beta(+\infty)=0 \\
D_{0^{+}}^{\delta-2} \beta(0) \geq \sum_{i=1}^{\infty} g\left(\xi_{i}\right) D_{0^{+}}^{\delta-1} u\left(\xi_{i}\right)+\lambda
\end{array}\right.
$$

Lemma 3.1 If $u \in E, D_{0^{+}}^{\delta} u \in L^{1}\left(\mathbb{R}^{+}\right)$and satisfies

$$
\left\{\begin{array}{l}
D_{0^{+}}^{\delta} u(t) \leq 0, \quad t \in(0,+\infty) \\
u(0)=D_{0^{+}}^{\delta-1} u(+\infty)=0, \quad D_{0^{+}}^{\delta-2} u(0) \geq \sum_{i=1}^{\infty} g\left(\xi_{i}\right) D_{0^{+}}^{\delta-1} u\left(\xi_{i}\right)
\end{array}\right.
$$

then $u(t) \geq 0$ for $t \in \mathbb{R}^{+}$.

Proof Let $-D_{0^{+}}^{\delta} u(t)=y(t) \geq 0$ for a.e. $t \in \mathbb{R}^{+}$, and $D_{0^{+}}^{\delta-2} u(0)=\sum_{i=1}^{\infty} g\left(\xi_{i}\right) D_{0^{+}}^{\delta-1} u\left(\xi_{i}\right)+\lambda$, then $\lambda \geq 0$.

According to Lemma 2.1, we know that the boundary value problem

$$
\left\{\begin{array}{l}
-D_{0^{+}}^{\delta} u(t)=y(t), \quad t \in(0,+\infty), \\
u(0)=D_{0^{+}}^{\delta-1} u(+\infty)=0, \\
D_{0^{+}}^{\delta-2} u(0)=\sum_{i=1}^{\infty} g\left(\xi_{i}\right) D_{0^{+}}^{\delta-1} u\left(\xi_{i}\right)+\lambda
\end{array}\right.
$$

has a unique solution

$$
u(t)=\int_{0}^{+\infty} G(t, s) y(s) \mathrm{d} s+\frac{\lambda}{\Gamma(\delta-1)} t^{\delta-2}
$$

From Lemma 2.2, we can obtain that $u(t) \geq 0$ for $t \in \mathbb{R}^{+}$. 
Theorem 3.2 Suppose (H1) and (H2) hold if boundary value problem (1.1) has a nonnegative lower solution $\alpha$ and an upper solution $\beta$ satisfies $\alpha(t) \leq \beta(t)$ for $t \in \mathbb{R}^{+}$. Then boundary value problem (1.1) has at least one positive solution $u$ that satisfies $\alpha(t) \leq u(t) \leq \beta(t)$ for $t \in \mathbb{R}^{+}$.

Proof Let

$$
F(t, u)= \begin{cases}f(t, \beta(t)), & u>\beta(t) \\ f(t, u), & \alpha(t) \leq u \leq \beta(t), \\ f(t, \alpha(t)), & u<\alpha(t)\end{cases}
$$

Since $f$ is an $L^{1}$-Carathéodory function, then $F$ is an $L^{1}$-Carathéodory function, too.

By Lemma 2.1, the boundary value problem

$$
\left\{\begin{array}{l}
D_{0^{+}}^{\delta} u(t)+q(t) F(t, u(t))=0, \quad t \in(0,+\infty), \\
u(0)=D_{0^{+}}^{\delta-1} u(+\infty)=0, \\
D_{0^{+}}^{\delta-2} u(0)=\sum_{i=1}^{\infty} g\left(\xi_{i}\right) D_{0^{+}}^{\delta-1} u\left(\xi_{i}\right)+\lambda
\end{array}\right.
$$

is equivalent to the integral equation

$$
u(t)=\int_{0}^{+\infty} G(t, s) q(s) F(s, u(s)) \mathrm{d} s+\frac{\lambda}{\Gamma(\delta-1)} t^{\delta-2} .
$$

Define the operator $Q: P \rightarrow P$ by

$$
(Q u)(t)=\int_{0}^{+\infty} G(t, s) q(s) F(s, u(s)) \mathrm{d} s+\frac{\lambda}{\Gamma(\delta-1)} t^{\delta-2} .
$$

For any $u \in P$ and $t \in \mathbb{R}^{+}$, by (H2), we can get

$$
0 \leq F(t, u(t)) \leq f(t, \beta(t))=f\left(t,\left(1+t^{\delta-1}\right) \frac{\beta(t)}{1+t^{\delta-1}}\right) \leq \varphi_{\|\beta\|}(t) .
$$

Let $\Omega_{1}=\{u \in P:\|u\| \leq R\}$, where the constant $R=\frac{L}{\Gamma(\delta)} \int_{0}^{+\infty} q(s) \varphi_{\|\beta\|}(s) \mathrm{d} s+\frac{\lambda}{\Gamma(\delta-1)}$.

Obviously, $\Omega_{1}$ is a closed and convex set. Then, for any $u \in \Omega_{1}$,

$$
\begin{aligned}
\frac{|Q u(t)|}{1+t^{\delta-1}} & =\left|\int_{0}^{+\infty} \frac{G(t, s)}{1+t^{\delta-1}} q(s) F(s, u(s)) \mathrm{d} s+\frac{\lambda}{\Gamma(\delta-1)} \frac{t^{\delta-2}}{1+t^{\delta-1}}\right| \\
& \leq \frac{L}{\Gamma(\delta)} \int_{0}^{+\infty} q(s) \varphi_{\|\beta\|}(s) \mathrm{d} s+\frac{\lambda}{\Gamma(\delta-1)}=R .
\end{aligned}
$$

That is, $\|Q u\| \leq R$, which implies $Q: \Omega_{1} \rightarrow \Omega_{1}$.

We can easily show that $Q$ is completely continuous since its proof is similar to Lemma 2.4.

According to the Schauder fixed point theorem, we know that $Q$ has at least one fixed point $u$. By Lemma 2.6, boundary value problem (3.1) has a positive solution $u$.

Next, we prove $\alpha(t) \leq u(t) \leq \beta(t)$ for $t \in \mathbb{R}^{+}$, and $u=u(t)$ is a solution of boundary value problem (1.1). 
Let $v(t)=u(t)-\alpha(t)$. According to $(\mathrm{H} 2)$, we get

$$
\begin{aligned}
& D_{0^{+}}^{\delta} \nu(t)=D_{0^{+}}^{\delta} u(t)-D_{0^{+}}^{\delta} \alpha(t)=-q(t) F(t, u(t))-D_{0^{+}}^{\delta} \alpha(t) \\
& \leq-q(t) F(t, u(t))+q(t) f(t, \alpha(t)) \leq 0, \\
& \nu(0)=u(0)-\alpha(0)=0, \\
& D_{0^{+}}^{\delta-1} \nu(+\infty)=D_{0^{+}}^{\delta-1} u(+\infty)-D_{0^{+}}^{\delta-1} \alpha(+\infty)=0,
\end{aligned}
$$

and

$$
\begin{aligned}
D_{0^{+}}^{\delta-2} \nu(0) & =D_{0^{+}}^{\delta-2} u(0)-D_{0^{+}}^{\delta-2} \alpha(0) \\
& \geq \sum_{i=1}^{\infty} g\left(\xi_{i}\right) D_{0^{+}}^{\delta-1} u\left(\xi_{i}\right)+\lambda-\sum_{i=1}^{\infty} g\left(\xi_{i}\right) D_{0^{+}}^{\delta-1} \alpha\left(\xi_{i}\right)-\lambda \\
& =\sum_{i=1}^{\infty} g\left(\xi_{i}\right) D_{0^{+}}^{\delta-1}\left(u\left(\xi_{i}\right)-\alpha\left(\xi_{i}\right)\right)=\sum_{i=1}^{\infty} g\left(\xi_{i}\right) D_{0^{+}}^{\delta-1} \nu\left(\xi_{i}\right) .
\end{aligned}
$$

From Lemma 3.1, we have $v(t) \geq 0$ for $t \in \mathbb{R}^{+}$, which implies that $u(t) \geq \alpha(t)$ for $t \in \mathbb{R}^{+}$. Similarly, we can show that $u(t) \leq \beta(t)$ for $t \in \mathbb{R}^{+}$.

Therefore, each solution $u$ of boundary value problem (3.1) satisfies $\alpha(t) \leq u(t) \leq \beta(t)$ for $t \in \mathbb{R}^{+}$. That is, $F(t, u(t))=f(t, u(t))$, and $u$ is a positive solution of boundary value problem (1.1).

\section{The properties of positive solutions}

Theorem 4.1 Assume (H1) and (H2) hold.

(1) If there exists a constant $\lambda=\bar{\lambda} \geq 0$ such that boundary value problem (1.1) has a positive solution $\bar{u}=\bar{u}(t)$, then for each $0 \leq \lambda \leq \bar{\lambda}$, boundary value problem (1.1) has a positive solution $u$ and $\frac{\lambda t^{\delta-2}}{\Gamma(\delta-1)} \leq u(t) \leq \bar{u}(t)$ for $t \in \mathbb{R}^{+}$.

(2) If there exists a constant $\lambda=\underline{\lambda} \geq 0$ such that boundary value problem (1.1) does not have positive solutions, then for each $\lambda \geq \underline{\lambda}$, boundary value problem (1.1) does not have positive solutions.

Proof (1) Since $\bar{u}=\bar{u}(t)$ is a positive solution of boundary value problem (1.1) with $\lambda=\bar{\lambda}$, then by Lemma 2.1,

$$
\bar{u}(t)=\int_{0}^{+\infty} G(t, s) q(s) f(s, \bar{u}(s)) \mathrm{d} s+\frac{\bar{\lambda} t^{\delta-2}}{\Gamma(\delta-1)} .
$$

Therefore, we can obtain that for any $0 \leq \lambda \leq \bar{\lambda}, \bar{u}(t) \geq \frac{\bar{\lambda} t^{\delta-2}}{\Gamma(\delta-1)} \geq \frac{\lambda t^{\delta-2}}{\Gamma(\delta-1)}, t \in \mathbb{R}^{+}$.

We take $\alpha=\frac{\lambda \delta^{\delta-2}}{\Gamma(\delta-1)}$ and $\beta=\bar{u}$, obviously, $\alpha \leq \beta$. We can easily show that $\alpha$ is a lower solution and $\beta$ is an upper solution of boundary value problem (1.1), respectively.

Then, according to Theorem 3.2, we can obtain that for any $0 \leq \lambda \leq \bar{\lambda}$, boundary value problem (1.1) has a positive solution $u$ and $\frac{\lambda \lambda^{\delta-2}}{\Gamma(\delta-1)} \leq u(t) \leq \bar{u}(t)$ for $t \in \mathbb{R}^{+}$.

(2) Assume that there exists a constant $\lambda_{0} \geq \underline{\lambda}$ such that boundary value problem (1.1) has a positive solution. In view of (1) in this theorem, for $\lambda=\underline{\lambda} \leq \lambda_{0}$, boundary value problem (1.1) has a positive solution, which is a contradiction. 
Denote

$$
\begin{aligned}
& f^{0}=\limsup _{u \rightarrow 0^{+}} \sup _{t \in \mathbb{R}^{+}} \frac{f\left(t,\left(1+t^{\delta-1}\right) u\right)}{u}, \quad f_{\infty}=\liminf _{u \rightarrow+\infty} \inf _{t \in\left[\frac{1}{k}, k\right]} \frac{f\left(t,\left(1+t^{\delta-1}\right) u\right)}{u}, \\
& \rho_{1}=\frac{\Gamma(\delta)}{\delta-1+L \int_{0}^{+\infty} q(s) \mathrm{d} s}, \quad \rho_{2}=\frac{4 k^{2}\left(1+k^{\delta-1}\right)^{2} \Gamma(\delta)}{\int_{\frac{1}{k}}^{k} q(s) \mathrm{d} s}
\end{aligned}
$$

Theorem 4.2 Suppose (H1) holds, if $f^{0}<\rho_{1}$, then there exists a constant $\lambda_{*} \geq 0$ such that boundary value problem (1.1) with $\lambda=\lambda_{*}$ has at least one positive solution.

Proof Because $f^{0}<\rho_{1}$, there exists a constant $r_{1}>0$ such that $f\left(t,\left(1+t^{\delta-1}\right) u\right)<\rho_{1} u \leq \rho_{1} r_{1}$ for any $t \in \mathbb{R}^{+}$and $u \in\left(0, r_{1}\right]$.

Set $B=\left\{u \in P:\|u\| \leq r_{1}\right\}$ and $0 \leq \lambda_{*} \leq \rho_{1} r_{1}$. Then, for any $u \in B$,

$$
f(s, u(s))=f\left(s,\left(1+s^{\delta-1}\right) \frac{u(s)}{1+s^{\delta-1}}\right) \leq \rho_{1} \frac{u(s)}{1+s^{\delta-1}} \leq \rho_{1} r_{1}
$$

and

$$
\begin{aligned}
\frac{T u(t)}{1+t^{\delta-1}} & =\int_{0}^{+\infty} \frac{G(t, s)}{1+t^{\delta-1}} q(s) f(s, u(s)) \mathrm{d} s+\frac{\lambda_{*}}{\Gamma(\delta-1)} \frac{t^{\delta-2}}{1+t^{\delta-1}} \\
& \leq \frac{L \rho_{1} r_{1}}{\Gamma(\delta)} \int_{0}^{+\infty} q(s) \mathrm{d} s+\frac{\lambda_{*}}{\Gamma(\delta-1)} \\
& \leq \frac{L \rho_{1} r_{1}}{\Gamma(\delta)} \int_{0}^{+\infty} q(s) \mathrm{d} s+\frac{\rho_{1} r_{1}}{\Gamma(\delta-1)} \\
& =\frac{\rho_{1} r_{1}}{\Gamma(\delta)}\left(L \int_{0}^{+\infty} q(s) \mathrm{d} s+\delta-1\right) \\
& =r_{1} .
\end{aligned}
$$

So $T(B) \subset B$. According to the Schauder fixed point theorem, we can obtain that $T$ has at least one fixed point on $B$. By Lemma 2.6, boundary value problem (1.1) has at least one positive solution.

Theorem 4.3 Suppose (H1) holds, if $f_{\infty}>\rho_{2}$, then there exist large enough positive constants $\hat{\lambda}$ such that boundary value problem (1.1) with $\lambda=\hat{\lambda}$ has no positive solution.

Proof Assume that there exists a constant $\hat{\lambda}>0$ and $\hat{\lambda}$ is large enough, boundary value problem (1.1) with $\lambda=\hat{\lambda}$ has a positive solution $\hat{u}=\hat{u}(t)$. By $f_{\infty}>\rho_{2}$, we know that

$$
f\left(t,\left(1+t^{\delta-1}\right) u\right)>\rho_{2} u
$$

for $t \in\left[\frac{1}{k}, k\right]$ and $u \geq r_{2}$, where constant $r_{2}>0$ is large enough.

Take $\hat{\lambda}>\Gamma(\delta-1)\left(1+k^{\delta-1}\right) r_{2}$. By Lemma 2.1,

$$
\hat{u}(t)=\int_{0}^{+\infty} G(t, s) q(s) f(s, \hat{u}(s)) \mathrm{d} s+\frac{\hat{\lambda}}{\Gamma(\delta-1)} t^{\delta-2},
$$


then

$$
\frac{\hat{u}\left(\frac{1}{k}\right)}{1+\left(\frac{1}{k}\right)^{\delta-1}} \geq \frac{\hat{\lambda}}{\Gamma(\delta-1)} \frac{\left(\frac{1}{k}\right)^{\delta-2}}{1+\left(\frac{1}{k}\right)^{\delta-1}}=\frac{\hat{\lambda}}{\Gamma(\delta-1)} \frac{k}{1+k^{\delta-1}}>\frac{\hat{\lambda}}{\Gamma(\delta-1)} \frac{1}{1+k^{\delta-1}}>r_{2},
$$

and $\|\hat{u}\|>r_{2}$. Then, for $s \in\left[\frac{1}{k}, k\right]$,

$$
f(s, \hat{u}(s))=f\left(s,\left(1+s^{\delta-1}\right) \frac{\hat{u}(s)}{1+s^{\delta-1}}\right)>\rho_{2} \frac{\hat{u}(s)}{1+s^{\delta-1}} .
$$

According to Lemma 2.5, we have $\min _{\frac{1}{k} \leq t \leq k} \frac{\hat{u}(t)}{1+t^{\delta-1}} \geq \frac{1}{4 k^{2}\left(1+k^{\delta-1}\right)}\|\hat{u}\|$. Then

$$
\begin{aligned}
\frac{\hat{u}\left(\frac{1}{k}\right)}{1+\left(\frac{1}{k}\right)^{\delta-1}} & =\int_{0}^{+\infty} \frac{G\left(\frac{1}{k}, s\right)}{1+\left(\frac{1}{k}\right)^{\delta-1}} q(s) f(s, \hat{u}(s)) \mathrm{d} s+\frac{\hat{\lambda}}{\Gamma(\delta-1)} \frac{\left(\frac{1}{k}\right)^{\delta-2}}{1+\left(\frac{1}{k}\right)^{\delta-1}} \\
& \geq \int_{\frac{1}{k}}^{k} \frac{G_{1}\left(\frac{1}{k}, s\right)}{1+\left(\frac{1}{k}\right)^{\delta-1}} q(s) f(s, \hat{u}(s)) \mathrm{d} s+\frac{\hat{\lambda}}{\Gamma(\delta-1)} \frac{k}{1+k^{\delta-1}} \\
& \geq \frac{\rho_{2}}{\Gamma(\delta)} \int_{\frac{1}{k}}^{k} \frac{\left(\frac{1}{k}\right)^{\delta-1}}{1+\left(\frac{1}{k}\right)^{\delta-1}} \frac{\hat{u}(s)}{1+s^{\delta-1}} q(s) \mathrm{d} s+\frac{\hat{\lambda}}{\Gamma(\delta-1)} \frac{1}{1+k^{\delta-1}} \\
& >\frac{\rho_{2}\|\hat{u}\|}{4 k^{2}\left(1+k^{\delta-1}\right)^{2} \Gamma(\delta)} \int_{\frac{1}{k}}^{k} q(s) \mathrm{d} s+r_{2} \\
& =\|\hat{u}\|+r_{2} .
\end{aligned}
$$

That is, $\|\hat{u}\|>\|\hat{u}\|+r_{2}$, which is a contradiction. Thus, there exist large enough constants $\hat{\lambda}>0$ such that boundary value problem (1.1) with $\lambda=\hat{\lambda}$ has no positive solution.

Theorem 4.4 Let $I \subset[0,+\infty)$ be a bounded set. Suppose (H1) holds, $f_{\infty}>\rho_{2}$, then for each $\lambda \in I$, there exists a constant $\tau>0$ such that $\|u\| \leq \tau$, where $u=u(t)$ is a solution of boundary value problem (1.1).

Proof Because $I$ is a bounded set, then for each $\lambda \in I$, there exists a constant $\sigma>0$ such that $0 \leq \lambda \leq \sigma$.

Since $f_{\infty}>\rho_{2}$, there exists a constant $r>0$ such that $f\left(t,\left(1+t^{\delta-1}\right) u\right)>\rho_{2} u$ for any $t \in\left[\frac{1}{k}, k\right]$ and $u \geq r$.

Let $\tau=4 k^{2}\left(1+k^{\delta-1}\right) r$. Assume that boundary value problem (1.1) has a solution $u=u(t)$ that satisfies $\|u\|>\tau$. Then

$$
\min _{\frac{1}{k} \leq t \leq k} \frac{u(t)}{1+t^{\delta-1}} \geq \frac{1}{4 k^{2}\left(1+k^{\delta-1}\right)}\|u\|>\frac{1}{4 k^{2}\left(1+k^{\delta-1}\right)} \tau=r .
$$

By Lemmas 2.1 and 2.5 , we have

$$
\begin{aligned}
\frac{u\left(\frac{1}{k}\right)}{1+\left(\frac{1}{k}\right)^{\delta-1}} & =\int_{0}^{+\infty} \frac{G\left(\frac{1}{k}, s\right)}{1+\left(\frac{1}{k}\right)^{\delta-1}} q(s) f(s, u(s)) \mathrm{d} s+\frac{\lambda}{\Gamma(\delta-1)} \frac{\left(\frac{1}{k}\right)^{\delta-2}}{1+\left(\frac{1}{k}\right)^{\delta-1}} \\
& \geq \int_{\frac{1}{k}}^{k} \frac{G_{1}\left(\frac{1}{k}, s\right)}{1+\left(\frac{1}{k}\right)^{\delta-1}} q(s) f(s, u(s)) \mathrm{d} s
\end{aligned}
$$




$$
\begin{aligned}
& \geq \frac{\rho_{2}}{\Gamma(\delta)} \int_{\frac{1}{k}}^{k} \frac{\left(\frac{1}{k}\right)^{\delta-1}}{1+\left(\frac{1}{k}\right)^{\delta-1}} \frac{u(s)}{1+s^{\delta-1}} q(s) \mathrm{d} s \\
& >\frac{\rho_{2}\|u\|}{4 k^{2}\left(1+k^{\delta-1}\right)^{2} \Gamma(\delta)} \int_{\frac{1}{k}}^{k} q(s) \mathrm{d} s=\|u\|,
\end{aligned}
$$

which is a contradiction.

So, for all solutions of boundary value problem (1.1), $u=u(t)$ satisfies that $\|u\| \leq \tau$.

\section{Existence, nonexistence and multiplicity of positive solutions}

Theorem 5.1 Suppose that (H1), (H2) hold, $f^{0}<\rho_{1}$ and $f_{\infty}>\rho_{2}$, then there exists a constant $\lambda^{*} \in(0,+\infty)$ such that the following results hold:

(1) Boundary value problem (1.1) has at least one positive solution for $\lambda=0$ and $\lambda=\lambda^{*}$;

(2) Boundary value problem (1.1) has at least two positive solutions for each $\lambda \in\left(0, \lambda^{*}\right)$;

(3) Boundary value problem (1.1) does not have any positive solutions for each $\lambda \in\left(\lambda^{*},+\infty\right)$.

Proof Let

$\Lambda=\{\lambda \in[0,+\infty)$ : the $\lambda$ such that boundary value problem (1.1)

has at least one positive solution\}.

Then by Theorem 4.2 we know that $\Lambda \neq \emptyset$.

In view of Theorem 4.1, $[0, \tilde{\lambda}] \subset \Lambda$ if and only if $\tilde{\lambda} \in \Lambda$.

According to $f_{\infty}>\rho_{2}$ and Theorem 4.3, we can show that $\Lambda$ is a bounded set. Let $\bar{M}=$ $\bigcup_{\tilde{\lambda} \in \Lambda}[0, \tilde{\lambda}]$, then $\bar{M}$ is a bounded set. Therefore, $\bar{M}$ has a supremum which is denoted by $\lambda^{*}=\sup \bar{M}>0$.

Next, we will prove that boundary value problem (1.1) has at least one positive solution for $\lambda=\lambda^{*}$.

Since $\lambda^{*}=\sup \bar{M}$, there exists a sequence $\left\{\lambda_{m}\right\} \subset \bar{M}$ that satisfies $\lambda_{m}<\lambda^{*}$ such that $\lambda_{m} \rightarrow$ $\lambda^{*}$ as $m \rightarrow+\infty$. Let $u_{m}(t)$ be the solution of boundary value problem (1.1) with $\lambda=\lambda_{m}$. In view of Lemma 2.1, we know that boundary value problem (1.1) with $\lambda=\lambda_{m}$ is equivalent to

$$
u_{m}(t)=\int_{0}^{+\infty} G(t, s) q(s) f\left(s, u_{m}(s)\right) \mathrm{d} s+\frac{\lambda_{m}}{\Gamma(\delta-1)} t^{\delta-2}, \quad m=1,2, \ldots
$$

According to Theorem 4.4, there exists a constant $\tau$ such that $\left\|u_{m}\right\| \leq \tau$, which implies that $\left\{u_{m}(t)\right\}$ is uniformly bounded. By Lemma 2.4 , we can easily show that $\left\{u_{m}(t)\right\}$ is equicontinuous. Then we know that $\left\{u_{m}(t)\right\}$ has a convergent subsequence, we assume that $\left\{u_{m}(t)\right\}$ itself converges uniformly to $u$ on $\mathbb{R}^{+}$, and $u \in P$. Since $f$ is an $L^{1}$-Carathéodory function, then by the Lebesgue dominated convergence theorem, as $m \rightarrow+\infty$, we have

$$
u(t)=\int_{0}^{+\infty} G(t, s) q(s) f(s, u(s)) \mathrm{d} s+\frac{\lambda^{*}}{\Gamma(\delta-1)} t^{\delta-2} .
$$

Hence, boundary value problem (1.1) has a positive solution $u$ for $\lambda=\lambda^{*}$. By Theorem 4.1, boundary value problem (1.1) has at least one positive solution for $\lambda \in\left[0, \lambda^{*}\right]$. And by 
the definition of $\lambda^{*}$ we know that boundary value problem (1.1) does not have positive solutions for each $\lambda \in\left(\lambda^{*},+\infty\right)$.

Finally, we prove that boundary value problem (1.1) has at least two positive solutions for $\lambda \in\left(0, \lambda^{*}\right)$.

For each $\lambda \in\left(0, \lambda^{*}\right)$, there exist $\underline{\lambda}, \bar{\lambda} \in \bar{M}$ such that $0<\underline{\lambda}<\lambda<\bar{\lambda}$. Let $\bar{u}, \underline{u}$ be the solutions of boundary value problem (1.1) for $\lambda=\bar{\lambda}, \lambda=\underline{\lambda}$, respectively. Then, according to Theorem 4.1, boundary value problem (1.1) has a positive solution $u_{1}=u_{1}(t)$ for $\lambda=\lambda_{1}$, and $\underline{u}(t) \leq u_{1}(t) \leq \bar{u}(t)$.

Let $\alpha=\underline{u}$ and $\beta=\bar{u}$. We can easily verify that $\alpha$ is a lower solution and $\beta$ is an upper solution of boundary value problem (1.1), and $\alpha(t)<\beta(t)$.

Choose $\hat{\lambda}>\lambda^{*}$ satisfies $\lambda<\lambda^{*}<\hat{\lambda}$.

We define $K:[\lambda, \hat{\lambda}] \times P \rightarrow E$ by

$$
K(r, u)=\int_{0}^{+\infty} G(t, s) q(s) f(s, u(s)) \mathrm{d} s+\frac{r}{\Gamma(\delta-1)} t^{\delta-2} .
$$

Let

$$
F(t, u)= \begin{cases}f(t, \beta(t)), & u>\beta(t), \\ f(t, u), & \alpha(t) \leq u \leq \beta(t) \\ f(t, \alpha(t)), & u<\alpha(t) .\end{cases}
$$

Define an integral operator $\widehat{K}:[\lambda, \hat{\lambda}] \times P \rightarrow E$ by

$$
\widehat{K}(r, u)=\int_{0}^{+\infty} G(t, s) q(s) F(s, u(s)) \mathrm{d} s+\frac{r}{\Gamma(\delta-1)} t^{\delta-2} .
$$

We can easily prove that $K$ and $\widehat{K}$ are completely continuous for each $r \in[\lambda, \hat{\lambda}]$ according to Lemma 2.4. In view of Lemma $2.6, u$ is a positive solution of boundary value problem (1.1) if and only if $u=K(\lambda, u)$.

By Theorem 4.4, there exists a constant $\tau$ such that the fixed point $u$ of $K$ satisfies $\|u\| \leq$ $\tau$ for each $r \in[\lambda, \hat{\lambda}]$. Let

$$
\Omega=\left\{u \in P:\|u\|<\tau, \alpha(t)<u(t)<\beta(t), t \in \mathbb{R}^{+}\right\} .
$$

Obviously, $\Omega \subset P$ is a nonempty open-bounded subset, then $u_{1} \in \Omega$.

Since $F$ is an $L^{1}$-Carathéodory function, then $F(t, u(t)) \leq \varphi_{\|\beta\|}(t)$. For any $(r, u) \in[\lambda, \hat{\lambda}] \times$ $P$, there exists a constant $R>\tau>0$ such that $\frac{\widehat{K}(r, u)}{1+t^{\delta-1}}<R$. Let $B(\theta, R)=\{u \in E:\|u\|<R\}$. Then $\Omega \subset P \cap B(\theta, R)$ and $u \neq \mu \widehat{K} u$ for $u \in P \cap \partial B(\theta, R)$ and any $\mu \in[0,1]$. Otherwise, if there exists $u_{0} \in P \cap \partial B(\theta, R)$ such that $u_{0}=\mu \widehat{K} u_{0}$, then $R=\left\|u_{0}\right\|=\mu\left\|\widehat{K} u_{0}\right\|<\mu R<R$, which is a contradiction. Hence, according to Lemma 2.7, for each $r \in[\lambda, \hat{\lambda}]$,

$$
i(\widehat{K}(r, u), P \cap B(\theta, R), P)=1 .
$$

Since $\widehat{K}$ does not have a fixed point on $P \cap(\overline{B(\theta, R)} \backslash \Omega)$, thus for any $r \in[\lambda, \hat{\lambda}]$,

$$
i(\widehat{K}(r, u), P \cap(B(\theta, R) \backslash \widehat{\Omega}), P)=0 .
$$


Since $\left.\widehat{K}\right|_{\Omega}=K$, then by the excision property of the fixed point index, we can obtain that for each $r \in[\lambda, \hat{\lambda}]$,

$$
\begin{aligned}
i(K(r, u), P \cap \Omega, P) & =i(\widehat{K}(r, u), P \cap \Omega, P) \\
& =i(\widehat{K}(r, u), P \cap B(\theta, R), P)-i(\widehat{K}(r, u), P \cap(B(\theta, R) \backslash \bar{\Omega}), P) \\
& =1 .
\end{aligned}
$$

Since $\hat{\lambda}>\lambda^{*}$, we know that boundary value problem (1.1) does not have positive solutions for each $\lambda \in\left(\lambda^{*},+\infty\right)$, then $K(\hat{\lambda}, u) \neq u$ for any $u \in P$. Hence,

$$
i(K(\hat{\lambda}, u), P \cap B(\theta, R), P)=0 .
$$

Define $H:[0,1] \times P \cap \overline{B(\theta, R)} \rightarrow E$ by

$$
H(\mu, u)=K((1-\mu) \lambda+\mu \hat{\lambda}, u)
$$

Obviously, $H$ is completely continuous.

We have $H(\mu, u) \neq u$ for $(\mu, u) \in[0,1] \times P \cap \partial B(\theta, R)$. Otherwise, if there exists $\left(\mu_{0}, u_{0}\right) \in$ $[0,1] \times P \cap \partial B(\theta, R)$ such that $H\left(\mu_{0}, u_{0}\right)=u_{0}$, then

$$
K\left(\left(1-\mu_{0}\right) \lambda+\mu_{0} \hat{\lambda}, u_{0}\right)=u_{0}, \quad u_{0} \in P,\left\|u_{0}\right\|=R .
$$

Therefore, $u_{0}=u_{0}(t)$ is a solution of boundary value problem (1.1) with $\lambda=\left(1-\mu_{0}\right) \lambda+\mu_{0} \hat{\lambda}$. Then $\left\|u_{0}\right\| \leq \tau$, which is a contradiction.

By (5.2) and the homotopy invariance of the fixed point index, we have

$$
\begin{aligned}
i(K(\lambda, u), P \cap B(\theta, R), P) & =i(H(0, u), P \cap B(\theta, R), P) \\
& =i(H(1, u), P \cap B(\theta, R), P) \\
& =i(K(\hat{\lambda}, u), P \cap B(\theta, R), P)=0 .
\end{aligned}
$$

According to (5.1), (5.3) and by using the additivity property of the fixed point index, we have

$$
i(K(\lambda, u), P \cap B(\theta, R) \backslash \bar{\Omega}, P)=-1 .
$$

Therefore, boundary value problem (1.1) has a solution $u_{2} \in P \cap B(\theta, R) \backslash \bar{\Omega}$. Because $u_{1} \in$ $\Omega$, we have $u_{1} \neq u_{2}$. Hence, boundary value problem (1.1) has at least two positive solutions for $\lambda \in\left(0, \lambda^{*}\right)$.

The proof is completed.

Definition 5.1 (see $[28,29]$ ) Suppose that $E$ is a Banach space, $P \subset E$ is a cone. We say that $\gamma$ is a nonnegative, continuous, concave functional on $P$ if $\gamma: P \rightarrow[0,+\infty)$ is continuous, and

$$
\gamma(\mu x+(1-\mu) y) \geq \mu \gamma(x)+(1-\mu) \gamma(y)
$$

for all $x, y \in P$ and $\mu \in[0,1]$. 
Set

$$
P_{c}=\{u \in P:\|u\|<c\}, \quad P(\gamma, a, b)=\{u \in P: a \leq \gamma(u),\|u\| \leq b\} .
$$

Lemma 5.2 (see [29]) Suppose that there exist constants $0<a<b<d \leq c, T: \bar{P}_{c} \rightarrow \bar{P}_{c}$ is a completely continuous operator, $\gamma: P \rightarrow[0,+\infty)$ is a continuous concave functional, for $u \in \bar{P}_{c}$, we have $\gamma(u) \leq\|u\|$, and the following conditions hold:

(C1) $\{u \in P(\gamma, b, d) \mid \gamma(u)>b\} \neq \emptyset$, and for $u \in P(\gamma, b, d), \gamma(T u)>b$;

(C2) for $u \in \bar{P}_{a}$, we have $\|T u\|<a$;

(C3) for $u \in P(\gamma, b, c)$ and $\|T u\|>d$, we have $\gamma(T u)>b$.

Then $T$ has at least three fixed points $u_{1}, u_{2}$ and $u_{3}$ satisfying

$$
\left\|u_{1}\right\|<a<\left\|u_{3}\right\|, \quad \gamma\left(u_{3}\right)<b<\gamma\left(u_{2}\right) .
$$

Theorem 5.3 Suppose (H1) holds. Let $0<a<b<d \leq c, \lambda<a \rho_{1}, b \rho_{2}<c \rho_{1}$ and suppose that $f$ satisfies the following conditions:

(H3) $f\left(t,\left(1+t^{\delta-1}\right) u\right)<a \rho_{1},(t, u) \in \mathbb{R}^{+} \times[0, a]$;

(H4) $f\left(t,\left(1+t^{\delta-1}\right) u\right)>b \rho_{2},(t, u) \in\left[\frac{1}{k}, k\right] \times[b, c]$;

(H5) $f\left(t,\left(1+t^{\delta-1}\right) u\right)<c \rho_{1},(t, u) \in \mathbb{R}^{+} \times[0, c]$.

Then boundary value problem (1.1) has at least three positive solutions $u_{1}, u_{2}$ and $u_{3}$ such that

$$
\left\|u_{1}\right\|<a, \quad b<\gamma\left(u_{2}\right), \quad a<\left\|u_{3}\right\| \quad \text { and } \quad \gamma\left(u_{3}\right)<b .
$$

Proof Define a nonnegative, continuous, concave functional on $E$ by

$$
\gamma(u)=\min _{\frac{1}{k} \leq t \leq k} \frac{u(t)}{1+t^{\delta-1}} .
$$

Next, we prove that the conditions of Lemma 5.2 hold.

For $u \in \bar{P}_{c}$, we have $\|u\| \leq c$, then for $t \in \mathbb{R}^{+}, 0 \leq \frac{u(t)}{1+t^{\delta-1}} \leq c$.

According to assumption (H5), we get

$$
f(s, u(s))=f\left(s,\left(1+s^{\delta-1}\right) \frac{u(s)}{1+s^{\delta-1}}\right)<c \rho_{1} .
$$

Hence,

$$
\begin{aligned}
\frac{T u(t)}{1+t^{\delta-1}} & =\int_{0}^{+\infty} \frac{G(t, s)}{1+t^{\delta-1}} q(s) f(s, u(s)) \mathrm{d} s+\frac{\lambda}{\Gamma(\delta-1)} \frac{t^{\delta-2}}{1+t^{\delta-1}} \\
& \leq \frac{L}{\Gamma(\delta)} c \rho_{1} \int_{0}^{+\infty} q(s) \mathrm{d} s+\frac{\lambda}{\Gamma(\delta-1)} \\
& \leq \frac{\rho_{1}}{\Gamma(\delta)}\left(L \int_{0}^{+\infty} q(s) \mathrm{d} s+\delta-1\right) c=c .
\end{aligned}
$$

Therefore, $T: \bar{P}_{c} \rightarrow \bar{P}_{c}$. According to Lemma 2.4, we have $T$ is completely continuous. Similarly, it follows from assumption (H3) that if $u \in \bar{P}_{a}$, we have $\|T u\| \leq a$. Condition (C2) of Lemma 5.2 holds. 
Let $u^{*}(t)=\frac{b+c}{2}\left(1+t^{\delta-1}\right), t \in \mathbb{R}^{+}$. We can show that $u^{*} \in P$ and $\left\|u^{*}\right\|=\frac{b+c}{2}<c$. According to the definition of $\gamma(u)$, we get $\gamma\left(u^{*}\right)=\frac{b+c}{2}>b$. Hence, $u^{*} \in\{u \in P(\gamma, b, d) \mid \gamma(u)>b\} \neq \emptyset$.

On the other hand, if $u \in P(\gamma, b, d)$, then $b \leq \min _{\frac{1}{k} \leq t \leq k} \frac{u(t)}{1+t^{\delta-1}}$ and $\|u\| \leq d \leq c$. Hence, for $t \in\left[\frac{1}{k}, k\right]$, we have $b \leq \frac{u(t)}{1+t^{\delta-1}} \leq c$. Then by assumption (H4), we get

$$
f(s, u(s))=f\left(s,\left(1+s^{\delta-1}\right) \frac{u(s)}{1+s^{\delta-1}}\right)>b \rho_{2} .
$$

Therefore,

$$
\begin{aligned}
\gamma(T u) & =\min _{\frac{1}{k} \leq t \leq k} \frac{T u(t)}{1+t^{\delta-1}} \\
& =\min _{\frac{1}{k} \leq t \leq k}\left(\int_{0}^{+\infty} \frac{G(t, s)}{1+t^{\delta-1}} q(s) f(s, u(s)) \mathrm{d} s+\frac{\lambda}{\Gamma(\delta-1)} \frac{t^{\delta-2}}{1+t^{\delta-1}}\right) \\
& \geq \int_{0}^{+\infty} \min _{\frac{1}{k} \leq t \leq k} \frac{G(t, s)}{1+t^{\delta-1}} q(s) f(s, u(s)) \mathrm{d} s \\
& \geq \frac{1}{4 k^{2}\left(1+k^{\delta-1}\right)} \int_{0}^{+\infty} \sup _{t \in \mathbb{R}_{+}} \frac{G(t, s)}{1+t^{\delta-1}} q(s) f(s, u(s)) \mathrm{d} s \\
& \geq \frac{1}{4 k^{2}\left(1+k^{\delta-1}\right)} \int_{\frac{1}{k}}^{k} \frac{G_{1}\left(\frac{1}{k}, s\right)}{1+\left(\frac{1}{k}\right)^{\delta-1}} q(s) f(s, u(s)) \mathrm{d} s \\
& >\frac{b \rho_{2}}{\Gamma(\delta) 4 k^{2}\left(1+k^{\delta-1}\right)} \int_{\frac{1}{k}}^{k} \frac{1}{1+k^{\delta-1}} q(s) \mathrm{d} s \\
& =\frac{b \rho_{2}}{\Gamma(\delta) 4 k^{2}\left(1+k^{\delta-1}\right)^{2}} \int_{\frac{1}{k}}^{k} q(s) \mathrm{d} s=b .
\end{aligned}
$$

Hence, for all $u \in P(\gamma, b, d)$, we have $\gamma(T u)>b$, which implies that condition (C1) of Lemma 5.2 holds.

Finally, for $u \in P(\gamma, b, c)$ and $\|T u\|>d$, we have $b \leq \min _{\frac{1}{k} \leq t \leq k} \frac{u(t)}{1+t^{\delta-1}}$ and $\|u\| \leq c$. Hence, $b \leq \frac{u(t)}{1+t^{\delta-1}} \leq c$ for $t \in\left[\frac{1}{k}, k\right]$. According to assumption (H4), we can obtain $\gamma(T u)>b$. Condition (C3) of Lemma 5.2 holds.

By Lemma 5.2, $T$ has at least three fixed points $u_{1}, u_{2}, u_{3}$ such that $\left\|u_{1}\right\|<a, b<\gamma\left(u_{2}\right)$, $a<\left\|u_{3}\right\|$ and $\gamma\left(u_{3}\right)<b$. These fixed points are positive solutions of (1.1).

\section{Examples}

To illustrate our main results, we present the following examples.

Example 6.1 We consider the infinite-point boundary value problem of nonlinear fractional differential equations on the infinite interval

$$
\left\{\begin{array}{l}
D_{0^{+}}^{\frac{8}{3}} u(t)+e^{-t-t^{\frac{5}{3}}} u^{2}=0, \quad t \in(0,+\infty) \\
u(0)=D_{0^{+}}^{\frac{5}{3}} u(+\infty)=0 \\
D_{0^{+}}^{\frac{2}{3}} u(0)=\sum_{i=1}^{\infty} \frac{1}{i^{2}} D_{0^{+}}^{\frac{5}{3}} u(i)+\lambda
\end{array}\right.
$$


where $\delta=\frac{8}{3}, q(t)=e^{-t}, f(t, u)=e^{-t^{\frac{5}{3}}} u^{2}$, it is easy to show that $(\mathrm{H} 2)_{5}$ holds. Let $g(t)=\frac{1}{t^{2}}$, $\xi_{i}=i$, then $\sum_{i=1}^{\infty} g\left(\xi_{i}\right)=\frac{\pi^{2}}{6}$ is convergent. Let $\varphi_{r}(t)=r^{2}\left(1+t^{\frac{5}{3}}\right)^{2} e^{-t^{\frac{5}{3}}} \in L^{1}\left(\mathbb{R}^{+}\right)$. We have $f\left(t,\left(1+t^{\frac{5}{3}}\right) u\right) \leq \varphi_{r}(t)$ for $u \leq r$ and $t \in \mathbb{R}^{+}$. Then $f$ is an $L^{1}$-Carathéodory function and $\int_{0}^{+\infty} q(s) \varphi_{r}(s) \mathrm{d} s \approx 1.088 r^{2}<+\infty$. Let $k=2$, we have $L=1+\frac{5}{3} \sum_{i=1}^{\infty} g\left(\xi_{i}\right)=1+\frac{5 \pi^{2}}{18}, \rho_{1}=$ $\frac{\Gamma\left(\frac{8}{3}\right)}{L+\frac{5}{3}} \approx 0.278, \rho_{2}=\frac{16 \Gamma\left(\frac{8}{3}\right)(1+2 \sqrt{2})^{2}}{\int_{\frac{1}{2}}^{2} e^{-s} \mathrm{~d} s} \approx 748.814$.

$$
f^{0}=\limsup _{u \rightarrow 0^{+}} \sup _{t \in \mathbb{R}^{+}} \frac{f\left(t,\left(1+t^{\frac{5}{3}}\right) u\right)}{u}=\limsup _{u \rightarrow 0^{+}} \sup _{t \in \mathbb{R}^{+}} \frac{e^{-t^{\frac{5}{3}}}\left(1+t^{\frac{5}{3}}\right)^{2} u^{2}}{u}=0<\rho_{1},
$$

and

$$
f_{\infty}=\liminf _{u \rightarrow+\infty} \inf _{t \in\left[\frac{1}{2}, 2\right]} \frac{f\left(t,\left(1+t^{\frac{5}{3}}\right) u\right)}{u}=\liminf _{u \rightarrow+\infty} \inf _{t \in\left[\frac{1}{2}, 2\right]} \frac{e^{-t^{\frac{5}{3}}}\left(1+t^{\frac{5}{3}}\right)^{2} u^{2}}{u}=+\infty>\rho_{2} .
$$

(1) Choose $r_{1}=0.18$, let $\lambda<\rho_{1} r_{1} \approx 0.05$. We can obtain $f\left(t,\left(1+t^{\frac{5}{3}}\right) u\right)<\rho_{1} r_{1}$ for $u \in(0,0.18], t \in \mathbb{R}^{+}$. By Theorems 4.1 and 4.2 , boundary value problem (6.1) has a positive solution for $\lambda \in[0,0.05]$.

(2) Choose $r_{2}=1,100$, let $\lambda>\Gamma\left(\frac{5}{3}\right)\left(1+2^{\frac{5}{3}}\right) r_{2} \approx 4,145.66$. We have $f\left(t,\left(1+t^{\frac{5}{3}}\right) u\right)>\rho_{2} r_{2}$ for $u \in[1,100,+\infty)$ and $t \in\left[\frac{1}{2}, 2\right]$. By Theorems 4.1 and 4.3 , boundary value problem (6.1) does not have a positive solution for $\lambda \in(4,200,+\infty)$.

(3) According to Theorem 5.1, we know that there exists a constant $\lambda^{*} \in(0.05,4,200)$ such that boundary value problem (6.1) has at least one positive solution for each $\lambda \in\left[0, \lambda^{*}\right]$, two positive solutions for any $\lambda \in\left(0, \lambda^{*}\right)$, and no positive solutions for $\lambda \in\left(\lambda^{*},+\infty\right)$.

Example 6.2 We consider the boundary value problem

$$
\left\{\begin{array}{l}
D_{0^{+}}^{\frac{5}{2}} u(t)+e^{-t} f(t, u(t))=0, \quad t \in(0,+\infty) \\
u(0)=D_{0^{+}}^{\frac{3}{2}} u(+\infty)=0 \\
D_{0^{+}}^{\frac{1}{2}} u(0)=\sum_{i=1}^{\infty} \frac{1}{(i-1) !} D_{0^{+}}^{\frac{3}{2}} u(i)+0.1
\end{array}\right.
$$

where

$$
f(t, u)= \begin{cases}e^{-\frac{t}{2}} \frac{u}{10\left(1+t^{\frac{3}{2}}\right)}, & 0 \leq u \leq 1, \\ e^{-\frac{t}{2}}\left(\frac{u}{10\left(1+t^{\frac{3}{2}}\right)}+10^{4}(u-1)\right), & 1 \leq u \leq 2, \\ e^{-\frac{t}{2}}\left(\frac{u}{10\left(1+t^{\frac{3}{2}}\right)}+10^{4}\right), & u \geq 2 .\end{cases}
$$

In this case, $\delta=\frac{5}{2}, q(t)=e^{-t}, g(t)=\frac{1}{(t-1) !}, \xi_{i}=i$. Then $\sum_{i=1}^{\infty} g\left(\xi_{i}\right)=e$ is convergent. Choose $\varphi_{r}(t)=\left(\frac{r\left(1+t^{\frac{3}{2}}\right)}{10}+10^{4}\right) e^{-\frac{t}{2}} \in L^{1}\left(\mathbb{R}^{+}\right)$. We have $f\left(t,\left(1+t^{\frac{3}{2}}\right) u\right) \leq \varphi_{r}(t)$ for $u \leq r$ and $t \in \mathbb{R}^{+}$. It is easy to show that $f$ is an $L^{1}$-Carathéodory function and $\int_{0}^{+\infty} q(s) \varphi_{r}(s) \mathrm{d} s \approx \frac{20,000}{3}+0.115 r<$ $+\infty$.

Take $a=1, b=2, c=d=10^{5}$ and $k=2$. We can get that $L=1+\frac{3}{2} \sum_{i=1}^{\infty} g\left(\xi_{i}\right)=1+1.5 e$, $\rho_{1}=\frac{\Gamma\left(\frac{5}{2}\right)}{L+\delta-1} \approx 0.202, \rho_{2}=\frac{16 \Gamma\left(\frac{5}{2}\right)(1+2 \sqrt{2})^{2}}{\int_{\frac{1}{2}}^{2} e^{-s} \mathrm{~d} s} \approx 661.601, \lambda=0.1<a \rho_{1} \approx 0.202$. 
Then the function $f$ satisfies

$$
\begin{aligned}
& f\left(t,\left(1+t^{\frac{3}{2}}\right) u\right)<\frac{1}{10}<a \rho_{1} \approx 0.202 \quad \text { for } t \in \mathbb{R}^{+}, 0 \leq u \leq 1 ; \\
& f\left(t,\left(1+t^{\frac{3}{2}}\right) u\right)>3,678.79>b \rho_{2} \approx 1,323.2 \quad \text { for } \frac{1}{2} \leq t \leq 2,2 \leq u \leq 10^{5} ; \\
& f\left(t,\left(1+t^{\frac{3}{2}}\right) u\right)<2 \times 10^{4}<c \rho_{1} \approx 2.021 \times 10^{4} \text { for } t \in \mathbb{R}^{+}, 0 \leq u \leq 10^{5} .
\end{aligned}
$$

Then, by Theorem 5.3, we know that boundary value problem (6.2) has at least three positive solutions $u_{1}, u_{2}$ and $u_{3}$ such that $\left\|u_{1}\right\|<1,2<\gamma\left(u_{2}\right), 1<\left\|u_{3}\right\|$ and $\gamma\left(u_{3}\right)<2$.

\section{Competing interests}

The authors declare that they have no competing interests.

\section{Authors' contributions}

The authors contributed equally to this paper. All authors read and approved the final manuscript.

\section{Acknowledgements}

The authors sincerely thank the anonymous reviewers and editor for their valuable suggestions, which improved the presentation of this paper. This work is supported by the National Natural Science Foundation of China (Grant No. 11171220) and the Hujiang Foundation of China (B14005).

\section{Publisher's Note}

Springer Nature remains neutral with regard to jurisdictional claims in published maps and institutional affiliations.

\section{Received: 30 November 2016 Accepted: 18 April 2017 Published online: 03 May 2017}

\section{References}

1. Kilbas, AA, Srivastava, HM, Trujillo, JJ: Theory and Applications of Fractional Differential Equations. North-Holland Mathematics Studies. Elsevier, Amsterdam (2006)

2. Miller, KS, Ross, B: An Introduction to the Fractional Calculus and Fractional Differential Equations. Wiley, New York (1993)

3. Zhou, Y: Basic Theory of Fractional Differential Equations. World Scientific, Singapore (2014)

4. Koeller, RC: Application of fractional calculus to the theory of viscoelasticity. J. Appl. Mech. 51, $299-307$ (1984)

5. Zhao, Y, Sun, S, Han, Z, Zhang, M: Positive solutions for boundary value problems of nonlinear fractional differential equations. Appl. Math. Comput. 217, 6950-6958 (2011)

6. Zhang, X, Han, Y: Existence and uniqueness of positive solutions for higher order nonlocal fractional differential equations. Appl. Math. Lett. 25, 555-560 (2012)

7. Zhao, X, Chai, C, Ge, W: Positive solutions for fractional four-point boundary value problems. Commun. Nonlinear Sci. Numer. Simul. 16, 3665-3672 (2011)

8. Ding, $X$, Feng, $Y, B$, R: Existence, nonexistence and multiplicity of positive solutions for nonlinear fractional differential equations. J. Appl. Math. Comput. 40, 371-381 (2012)

9. Bai, Z: Solvability for a class of fractional m-point boundary value problem at resonance. Comput. Math. Appl. 62 , 1292-1302 (2011)

10. Bai, Z, Zhang, Y: Solvability of fractional three-point boundary value problems with nonlinear growth. Appl. Math. Comput. 218, 1719-1725 (2011)

11. Deng, J, Ma, L: Existence and uniqueness of solutions of initial value problems for nonlinear fractional differential equations. Appl. Math. Lett. 23, 676-680 (2010)

12. Liu, X, Jia, M, Ge, W: The method of lower and upper solutions for mixed fractional four-point boundary value problem with p-Laplacian operator. Appl. Math. Lett. 65, 56-62 (2017)

13. Liu, X, Jia, M: Multiple solutions for fractional differential equations with nonlinear boundary conditions. Comput. Math. Appl. 59, 2880-2886 (2010)

14. Su, X, Jia, M, Li, M: The existence and nonexistence of positive solutions for fractional differential equations with nonhomogeneous boundary conditions. Adv. Differ. Equ. 2016, 30 (2016)

15. Yang, L: Existence results for higher order fractional differential equation with multi-point boundary condition. Bound. Value Probl. 2012, 57 (2012)

16. Zhang, $X$ : Positive solutions for a class of singular fractional differential equation with infinite-point boundary value conditions. Appl. Math. Lett. 39, 22-27 (2015)

17. Dong, X, Bai, Z, Zhang, S: Positive solutions to boundary value problems of $p$-Laplacian with fractional derivative. Bound. Value Probl. 2017, 5 (2017)

18. Bai, Z, Zhang, S, Sun, S, Yin, C: Monotone iterative method for a class of fractional differential equations. Electron. J. Differ. Equ. 2016, 6 (2016)

19. Jia, M, Liu, X: Multiplicity of solutions for integral boundary value problems of fractional differential equations with upper and lower solutions. Appl. Math. Comput. 232, 313-323 (2014) 
20. Bai, Z: Eigenvalue intervals for a class of fractional boundary value problem. Comput. Math. Appl. 64, 3253-3257 (2012)

21. Liang, S, Zhang, J: Existence of three positive solutions of $m$-point boundary value problems for some nonlinear fractional differential equations on an infinite interval. Comput. Math. Appl. 61, 3343-3354 (2011)

22. Shen, $\mathrm{C}, \mathrm{Zhou}, \mathrm{H}$, Yang, L: On the existence of solution to a boundary value problem of fractional differential equation on the infinite interval. Bound. Value Probl. 2015, 241 (2015)

23. Li, X, Liu, X, Jia, M, Li, Y, Zhang, S: Existence of positive solutions for integral boundary value problems of fractional differential equations on infinite interval. Math. Methods Appl. Sci. 40, 1892-1904 (2017)

24. Liu, Y: Existence and unboundedness of positive solutions for singular boundary value problems on half-line. Appl. Math. Comput. 144, 543-556 (2003)

25. Chen, Y, Tang, X: Positive solutions of fractional differential equations at resonance on the half-line. Bound. Value Probl. 2012, 64 (2012)

26. Shah, $\mathrm{SAH}$, Rehman, $\mathrm{MU}$ : A note on terminal value problems for fractional differential equations on infinite interval. Appl. Math. Lett. 52, 118-125 (2016)

27. Kou, $\mathrm{C}, \mathrm{Zhou}, \mathrm{H}, \mathrm{Yan}, \mathrm{Y}$ : Existence of solutions of initial value problems for nonlinear fractional differential equations on the half-axis. Nonlinear Anal. 74, 5975-5986 (2011)

28. Guo, D, Lakshmikanthan, V: Nonlinear Problems in Abstract Cones. Notes and Reports in Mathematics in Science and Engineering, vol. 5. Academic Press, New York (1988)

29. Leggett, RW, Williams, LR: Multiple positive fixed points of nonlinear operators on ordered Banach spaces. Indiana Univ. Math. J. 28, 673-688 (1979)

\section{Submit your manuscript to a SpringerOpen ${ }^{\circ}$ journal and benefit from:}

- Convenient online submission

- Rigorous peer review

Immediate publication on acceptance

- Open access: articles freely available online

- High visibility within the field

- Retaining the copyright to your article 